\title{
ON THE EFFECTS OF QUANTIZATION ON MISMATCHED PULSE COMPRESSION FILTERS DESIGNED USING L-p NORM MINIMIZATION TECHNIQUES
}

\author{
J.E. Cilliers, J.C. Smit \\ Council for Scientific and Industrial Research, South Africa, <jcilliers@csir.co.za>, Fax: +27 128412455
}

Keywords: Pulse compression, sidelobe reduction, mismatched filtering, L-p Norm, quantization

\begin{abstract}
In [1] the authors introduced a technique for generating mismatched pulse compression filters for linear frequency chirp signals. The technique minimizes the sum of the pulse compression sidelobes in an $L_{p}$-norm sense. It was shown that extremely constant sidelobe levels (better than $60 \mathrm{~dB}$ ) can be achieved for minimal mismatch loss and broadening of the compression peak. This paper reports on an investigation into the effect of quantization on pulse compression filters designed using the abovementioned technique. Simulation results for 8-bit and 16-bit implementations of a pulse compressor system are presented.
\end{abstract}

\section{Introduction}

Pulse compression gives radar designers the ability to obtain sufficient energy on a target for target detection without decreasing the range resolution of the system or resorting to the use of very high power transmitters. Pulse compression thus permits the use of lower power transmitters with longer pulse lengths to maintain the energy content of a pulse. A matched filter is used on reception to maximize the signal to noise ratio of the received signal [3]. The actual transmitted waveforms are chosen so as to have an Autocorrelation Function (ACF) with a narrow peak at zero time shift and sidelobe values as low as possible at all other times. The sidelobes have the undesirable effect of masking smaller targets in close proximity to larger targets, such as clutter returns.

No direct design techniques exist for the generation of pulse compression waveforms with optimally low sidelobe levels. This has resulted in several approaches to the problem of designing "good" pulse compression waveforms. Early pulse compression systems were based on linear frequency modulated waveforms [4], [5] so several techniques have been developed to reduce the sidelobe levels of this broad class of pulse compression waveforms. These include amplitude windowing of the signal and the dual thereof which is frequency windowing. Notably De Witte and Griffiths [6] achieved sidelobe levels of approximately $70 \mathrm{~dB}$ by using non-linear frequency chirp waveforms.

Optimal binary phase shift codes have been found by means of exhaustive search techniques [7],[8],[9],[10]. Searches for good quadriphase codes have been reported in [11] and [12]. Gartz [13] and Nunn [14] have addressed the search for polyphase codes. Code searches are constrained by the computational complexity of the search process, which limits the lengths of codes which can be found using this technique. This has lead some authors to develop techniques for constructing codes which have close to optimal sidelobe levels. The Frank codes [15] and P(n,k) codes developed by Felhauer [16] are well known codes in this category.

Post-processing of the pulse compressor output such as sidelobe cancellation [17] and sidelobe smoothing [18] have also been reported.

Alternatively the pulse compression filter can be deliberately mismatched to reduce the sidelobe levels, but this implies a loss in signal to noise ratio and broadening of the compression peak as reported in [1]. An overview of the sidelobe reduction technique presented in [1] is given in the following section.

\section{Sidelobe level reduction by application of norm minimization to the sidelobes}

The mismatched pulse compression filter can be designed by minimizing the sum of the magnitudes of the complex sidelobe values ( $L_{2}-$ norm). This leads to the minimization of the energy in the sidelobes, whereas minimization of the $L_{\infty}-$ norm will minimize the peak sidelobe level. The $L_{\infty}-$ norm is not a well behaved function, so $L_{p}$-norms with $p=2 P$ were used in [1] and the solutions were found by means of numerical techniques.

Given the discrete time transmit sequence $\left\{a_{n}\right\}$ and filter coefficients $\left\{x_{n}\right\}$, the output of the filter is given by

$$
b_{i}=\sum_{k} a_{i+1-k} h_{k}
$$

where for the matched filter case $h_{n}=a_{N-n}^{*}$. The output of the 
pulse compression filter can be written in matrix form as

$$
\mathbf{b}=\mathbf{A}_{\mathbf{F}} \mathbf{x}
$$

where

$$
\begin{aligned}
& \mathbf{b}=\left[\begin{array}{llll}
b_{1} & b_{2} & \cdots & b_{2 N-1}
\end{array}\right]^{T}, \\
& \mathbf{x}=\left[\begin{array}{llll}
x_{1} & x_{2} & \cdots & x_{N}
\end{array}\right]^{T}
\end{aligned}
$$

and

$$
\mathbf{A}_{\mathbf{F}}=\left[\begin{array}{cccccccc}
a_{1} & a_{2} & \cdots & a_{N} & 0 & 0 & \cdots & 0 \\
0 & a_{1} & a_{2} & \cdots & a_{N} & 0 & \cdots & 0 \\
\vdots & \ddots & \ddots & \ddots & \ddots & \ddots & \ddots & \vdots \\
0 & \cdots & 0 & a_{1} & a_{2} & \cdots & a_{N} & 0 \\
0 & \cdots & 0 & 0 & a_{1} & a_{2} & \cdots & a_{N}
\end{array}\right]^{T}
$$

The above formulation leads to the following convenient expression for the sum-of-squares of the convolution sequence:

$$
\mathbf{b}^{H} \mathbf{b}=\left\|b_{1}\right\|^{2}+\left\|b_{2}\right\|^{2}+\cdots+\left\|b_{2 N-1}\right\|^{2} .
$$

The sidelobe measure function can now be formulated by defining a new matrix $\mathbf{A}$ which is similar to $\mathbf{A}_{\mathbf{F}}$, except that the rows in $\mathbf{A}_{\mathbf{F}}$ which produce the compression peak are removed.

The method of Lagrange multipliers [2] is used to find a solution for $\mathbf{X}$ that will minimize the sidelobe measure cost function while satisfying the constraint that a pulse compression peak with amplitude $b_{\text {peak }}$ must be produced.

The analytical solution for the $L_{2}$ case is given by

$$
\mathbf{x}=\frac{b_{p e a k} \mathbf{C}^{-1} \mathbf{a}^{H}}{\mathbf{a} \mathbf{C}^{-1} \mathbf{a}^{H}}
$$

where

$$
\mathbf{C}=\mathbf{A}^{H} \mathbf{A} .
$$

Numerical techniques had to be used to solve the $L_{2 P}$-norm case. The following set of simultaneous equations have to be solved together with the constraint given in Equation (10).

$$
\begin{aligned}
& \frac{\sum_{i=1}^{2 N-1}\left(\left[\mathbf{x}^{H} \mathbf{C}_{i} \mathbf{x}\right]^{P-1} \mathbf{C}_{i} \mathbf{x}\right)}{\left(\sum_{i=1}^{2 N-1}\left(\left[\mathbf{x}^{H} \mathbf{C}_{i} \mathbf{x}\right]^{P}\right)\right)^{1-\frac{1}{2 P}}+\lambda \mathbf{a}^{H}=\mathbf{0},} \\
& g(\mathbf{x})=\mathbf{a x}-b_{\text {peak }}=0 .
\end{aligned}
$$

An example of a solution for a linear frequency chirp transmit pulse with a time-bandwidth product (TBWP) of 50 is given in Figure 1.

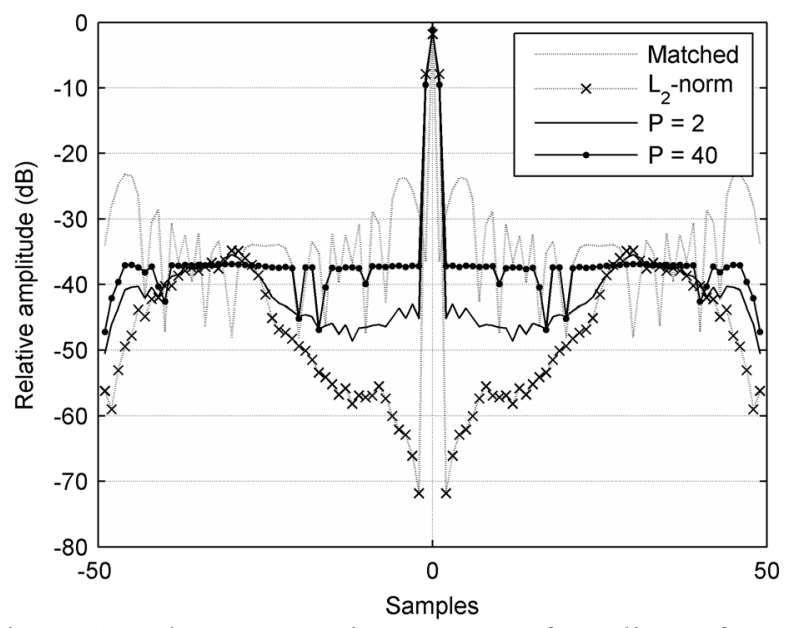

Figure 1: Pulse compression response for a linear frequency chirp transmit pulse with a TBWP of 50.

\subsection{Additional non-zero coefficients}

To reduce the sidelobe level further, the pulse compression filter was symmetrically extended in time to be longer than the transmitted pulse. The extra coefficients are referred to as additional non-zero coefficients (ANZC). An example of the result achieved using 100 ANZC (i.e. 50 before and 50 after the standard matched filter length) is shown in Figure 2. By comparing Figure 1 and Figure 2 it can be seen that the ANZC's have allowed the sidelobe level to be significantly reduced.

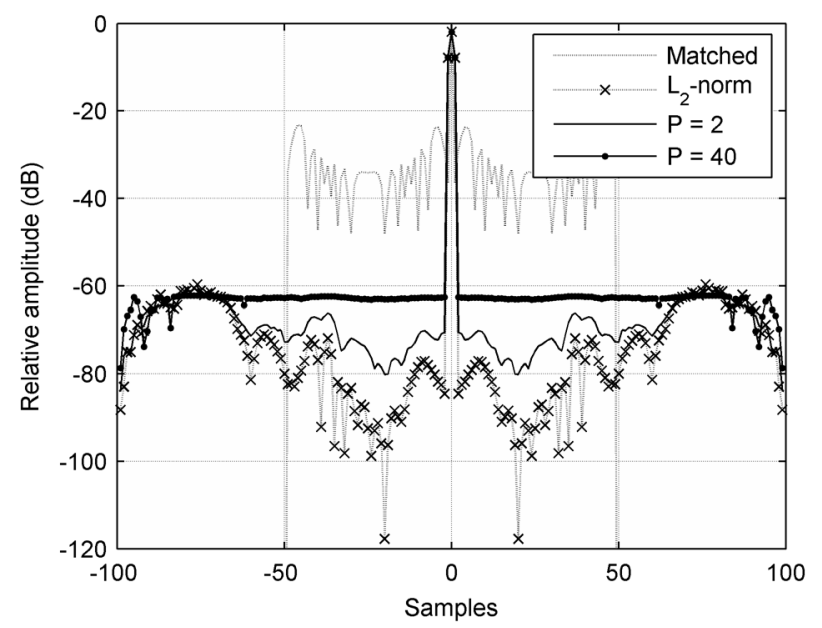

Figure 2: Pulse compression response for a linear frequency chirp transmit pulse with a TBWP of 50 and 100 ANZC's.

\section{Effect of coefficient quantization}

Most radar systems utilise fixed point arithmetic in the implementation of signal processing algorithms due to realtime processing constraints. This prompted the authors to investigate the effect of coefficient quantization on the 
extremely low sidelobe levels obtained in [1], an example of which is shown in Figure 2. The transmit and receive waveforms were quantized to 8 and 16 bits and the pulse compressor outputs were simulated with the assumption that all bit growth was retained in the FIR filter implementation. Some example results are depicted in the figures that follow. For all the figures the transmit waveform was a linear frequency chirp with a TBWP of 50 .

\subsection{Results with no additional non-zero coefficients}

In this subsection three figures are presented showing the output of the optimized pulse compression filter as well as that of the matched filter for reference. No ANZC's were used and $\mathrm{P}$ was set to 1, 2 and 40.

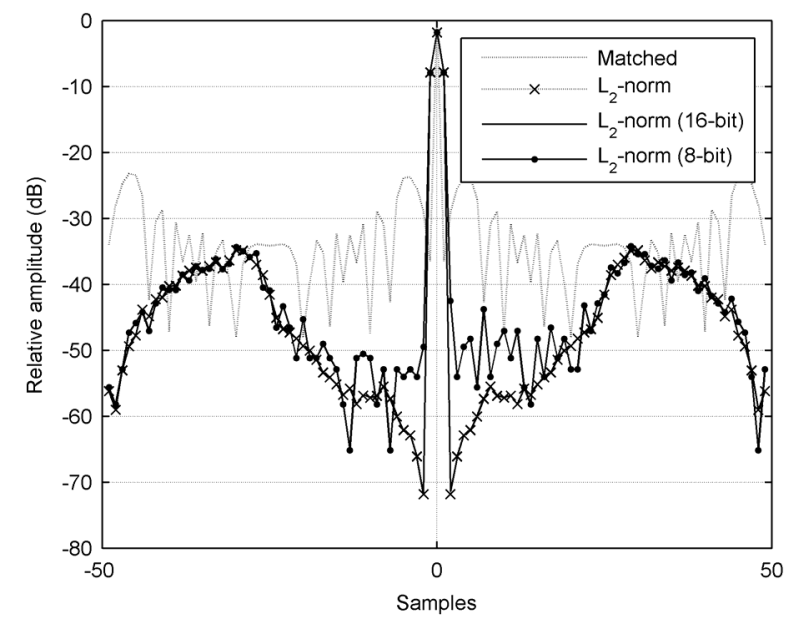

Figure 3: Comparison of sidelobe responses for receive filters with $\mathrm{P}=1$ and $\mathrm{ANZC}=0$.

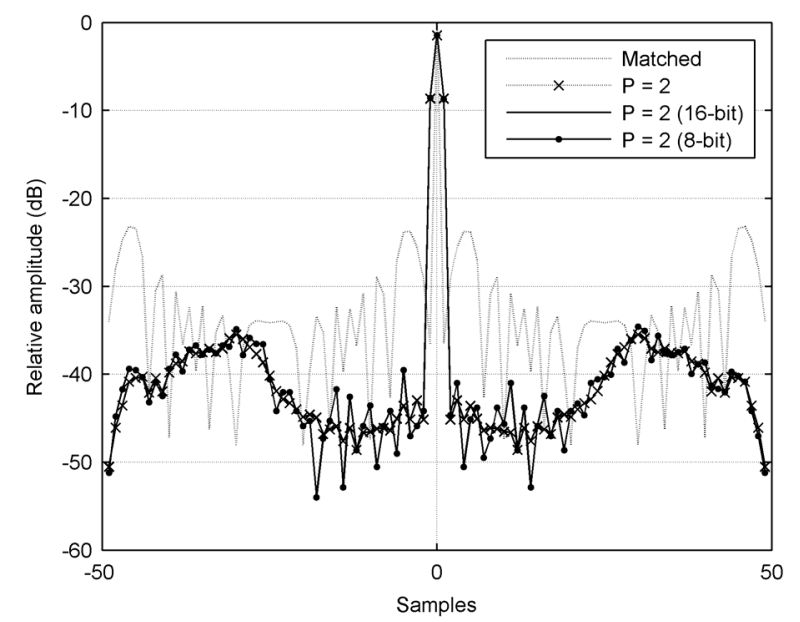

Figure 4: Comparison of sidelobe responses for receive filters with $\mathrm{P}=2$ and $\mathrm{ANZC}=0$.

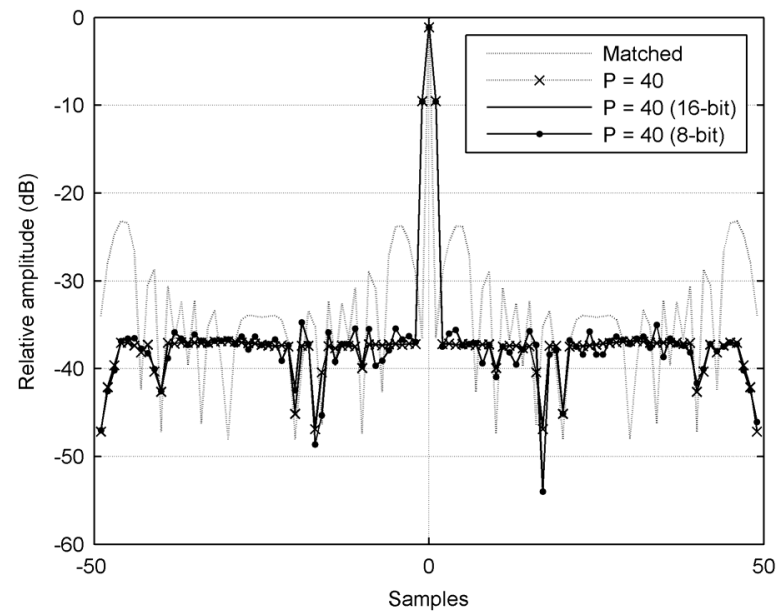

Figure 5: Comparison of sidelobe responses for receive filters with $\mathrm{P}=40$ and $\mathrm{ANZC}=0$.

The results presented in this subsection show that 16-bit quantization has a negligible effect on the resulting sidelobe response as compared to the floating point version of the filter (the two traces are indistinguishable). The 8-bit quantization adds a small amount of high frequency noise to the sidelobe response, approximately $45 \mathrm{~dB}$ below the pulse compression peak. This degradation in achievable sidelobe level should be tolerable for many applications.

\subsection{Results using additional non-zero coefficients}

In this subsection three more figures are presented showing the output of the optimized pulse compression filter as well as that of the matched filter for reference. For these figures 100 ANZC's were used and $\mathrm{P}$ was set to 1,2 and 40.

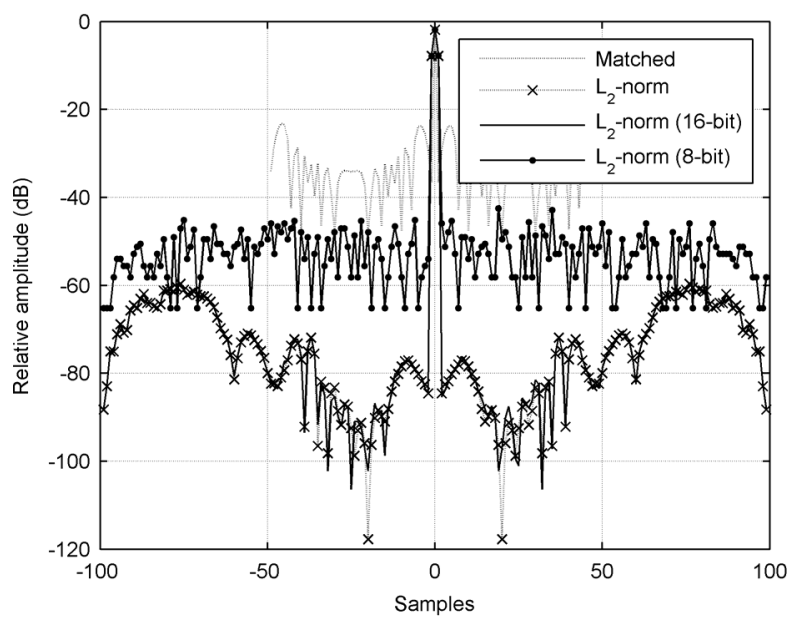

Figure 6: Comparison of sidelobe responses for receive filters with $\mathrm{P}=1$ and $\mathrm{ANZC}=100$. 


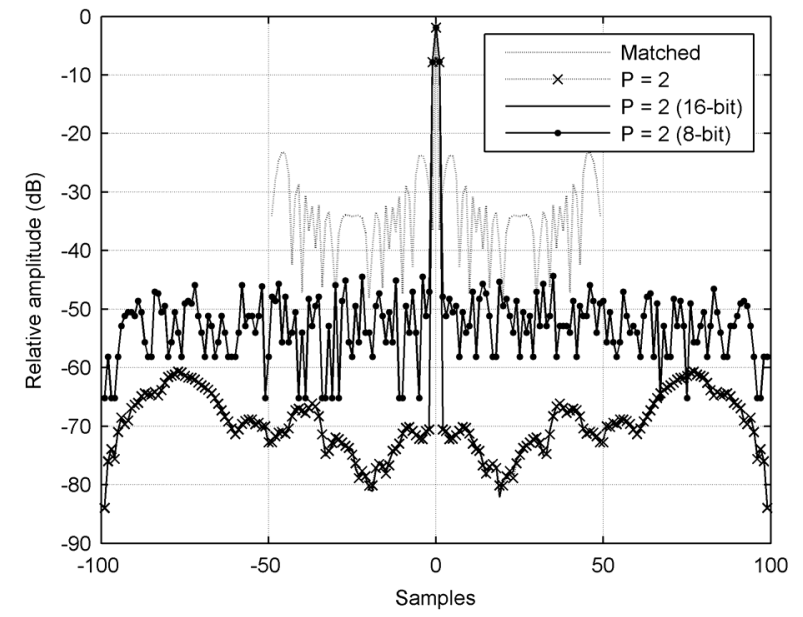

Figure 7: Comparison of sidelobe responses for receive filters with $\mathrm{P}=2$ and $\mathrm{ANZC}=100$.

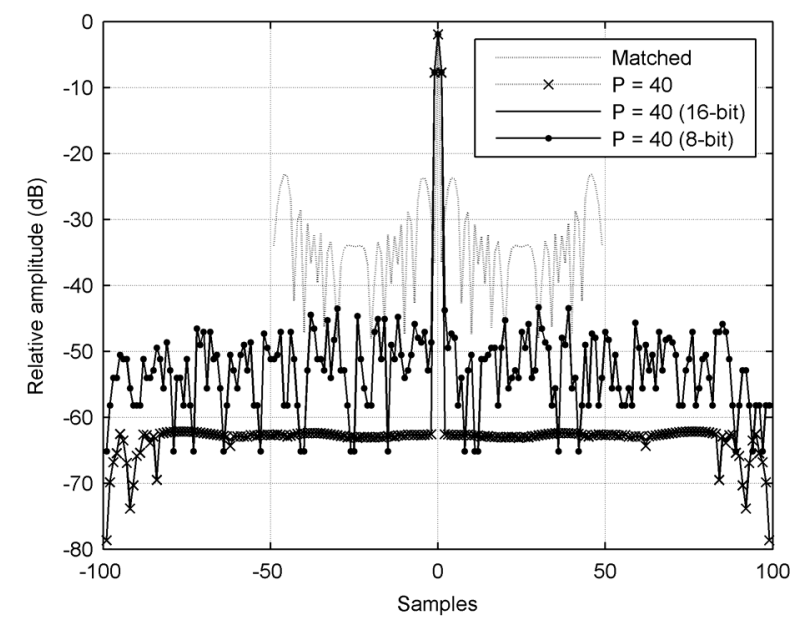

Figure 8: Comparison of sidelobe responses for receive filters with $\mathrm{P}=40$ and $\mathrm{ANZC}=100$.

The results presented in this subsection show that 16-bit quantization has negligible effect on the resulting sidelobe response as compared to the floating point version of the filter. The only exception to this is the sidelobe levels in Figure 6 which are below $-100 \mathrm{~dB}$, which would be inconsequential given the $-60 \mathrm{~dB}$ peak sidelobe level.

\section{Discussion}

This paper has shown that the achievable sidelobe level for mismatched pulse compression filters designed using the minimization of $L_{p}$-norms is relatively insensitive to quantization of the filter coefficients and transmit coefficients. It was shown that 16-bit quantized filters produce sidelobe levels which match the floating point versions very well. Filters with no ANZC's are relatively insensitive to 8-bit quantization.

The use of 8-bit quantization for filters with 100 ANZC's however limits the achievable sidelobe level to approximately $-55 \mathrm{~dB}$. This represents an increase of at least $7 \mathrm{~dB}$ in sidelobe level for the floating point case. It might be possible to improve this figure if use is made of optimization algorithms which have been designed to search discrete spaces. This is a topic for further research.

\section{Acknowledgements}

This research was partially funded by the South African Department of Science and Technology.

\section{References}

[1] J.E. Cilliers and J.C. Smit, "Pulse compression sidelobe reduction by minimization of $\mathrm{L}_{\mathrm{p}}$-norms," to appear in IEEE Transactions on Aerospace and Electronic Systems.

[2] T. K. Moon and W. C. Stirling, Mathematical methods and algorithms for signal processing, Prentice Hall: New Jersey, 2000.

[3] D. O. North, "An analysis of the factors which determine signal-noise discrimination in pulsed carrier systems," RCA Lab., Rep. PTR-6C; reprinted in Proc. IEEE, 51, pp. 1016-1027, July 1963.

[4] C. E. Cook, "The early history of pulse compression radar-the history of pulse compression at Sperry Gyroscope Company," IEEE Trans. Aerospace and Electronic Systems, vol. 24, no. 6, pp. 825-833, November 1988.

[5] W. M. Siebert, "The early history of pulse compression radar-the development of AN/FPS-17 coded-pulse radar at Lincoln Laboratory," IEEE Trans. Aerospace and Electronic Systems, vol. 24, no. 6, pp. 833-837, November 1988.

[6] E. De Witte, H. D. Griffiths, "Improved ultra-low range sidelobe pulse compression waveform design," IEE Electronic Letters, vol. 40, no. 22, pp 1448-1450, October 2004.

[7] J. Lindner, "Binary sequences up to length 40 with best possible autocorrelation function," IEEE Proceedings, vol. 11, no. 21, October 1975.

[8] M. N. Cohen, M. R. Fox and J. M. Baden, "Minimum peak sidelobe pulse compression codes," Conf. Rec. IEEE 1990 International Radar Conf., pp 633-638.

[9] S. Mertens, "Exhuastive search for low autocorrelation binary sequences," Journal of Physics A: Math. Gen., vol 29, pp L473-L481, 1996.

[10] G. Coxson and J. Russo, "Efficient exhaustive search for optimal-peak-sidelobe binary codes," IEEE Trans. Aerospace and Electronic Systems, vol. 41, no. 1, pp. 302-308, January 2005.

[11] W. H. Mow, "Best quadriphase codes up to length 24," IEE Electronic Letters, Vol. 29, No. 10, pp 923-925, May 1993.

[12] H. D. Luke, H. D. Schotten, H. Hadinejad-Mahram, "Binary and quadriphase sequences with optimal autocorrelation properties: a survey," IEEE Trans. Information Theory, vol. 49, no. 12, pp 3271-3282, December 2003. 
[13] K. J. Gartz, "Generation of uniform amplitude complex code sets with low correlation sidelobes," IEEE Trans. Signal Processing, vol. 40, no. 2, pp 343-351, February 1992.

[14] C. Nunn, "Constrained optimization applied to pulse compression codes, and filters," Conf. Rec. IEEE 2005 International Radar Conf., pp 190-194.

[15] R. Frank, "Polyphase complementary codes," IEEE Trans. Information Theory, vol. 26, no. 6, pp 641-647, November 1980.

[16] T. Felhauer, "Design and analysis of new $p(n, k)$ polyphase pulse compression codes," IEEE Trans. Aerospace and Electronic Systems, vol. 30, no. 3, pp 865-874, July 1994.

[17] W. K. Lee, H. D. Griffiths and R. Benjamin, "Integrated sidelobe energy reduction technique using optimal polyphase codes," IEE Letters, Vol. 35, No. 4, pp 20902091, November 1999.

[18] R. Sato and M. Shinriki, "Simple mismatched filter for binary pulse compression code with small PSL and small S/N loss," IEEE Trans. Aerospace and Electronic Systems, Vol. 39, No. 2, pp 711-718, April 2003. 